\title{
Cognitive self-mediation training with hyperkinetic children
}

\author{
SAMUEL F. MOORE and SHERWOOD O. COLE \\ Rutgers University, Camden, New Jersey 08102
}

\begin{abstract}
A group of hyperkinetic children from an area residential and educational facility was trained to verbally mediate behavior on problem-solving tasks by undergraduate volunteers. Such training involved the initial modeling of appropriate task behavior by the trainer and, by selective cuing and reinforcement, the child's eventual imitation of the trainer-modeled behavior on the same tasks. Relative to time and treatment controls, the hyperkinetic children demonstrated significant changes on a number of measures of cognitive abilities. Both the value and limitation of generalizing such findings are briefly discussed.
\end{abstract}

Among the deficiencies in attentional processes demonstrated by hyperkinetic children is their inability to delay initial impulses in the interest of carefully examining and reflecting upon alternative choices on discrimination tasks, and failures in the selective perception of figures independently of the context in which they are presented (Campbell, Douglas, \& Morgenstern, 1971; Cohen, Weiss, \& Minde, 1972). These inefficiencies in the modulation of attentional processes are demonstrated across a wide range of cognitive, emotional, and social behavior (Douglas, 1975) and are undoubtedly a contributing factor in hyperkinetics' generally less than successful response in regular classroom academics (Alabiso, 1972). The most prevalent treatment for hyperkinesis, drug therapy, has equivocal effects at best on these attentional deficiencies (Douglas, 1975), and drug treatment's potentially negative side effects, particularly when applied over a prolonged period, raise further questions regarding its efficacy (Cole \& Moore, 1976; Douglas, 1975). Consequently, alternative methods of modifying attentional deficiencies remain a critical need in the treatment of hyperkinesis.

A modeling procedure termed cognitive selfinstructional (CSI) training has been successful in modifying similar inefficiencies in attentional processes for impulsive children (Meichenbaum \& Goodman, 1971). Particularly noteworthy is the degree to which CSI training effects generalize across a number of cognitive tasks for the impulsive children. In application to hyperkinetics, the CSI procedure offers the promise of affecting several of their inefficiencies in attentional processes simultaneously. An additional advantage of the procedure is its highly structured nature, which suggests that it might be successfully applied by paraprofessionals.

The present investigation examines the efficacy of a CSI training program applied by undergraduate volunteers to a sample of hyperkinetic children from a residential special education facility.

\section{METHOD}

\section{Subjects}

The study population consisted of three females and 11 males between the ages of 8 and 12 years (mean: 9 years, 4 months) selected from an area educational facility for children with behavioral and educational problems of sufficient severity to warrant residential placement. The school's screening and diagnostic judgments of hyperkinesis were accepted as the initial criterion for defining the study population. No child with a clearly documented neurological impairment or an IQ below 85 was included. For practical purposes, there was no interruption of stimulant medication during the course of the study for any child receiving such treatment.

\section{Procedure}

Six advanced undergraduate psychology majors were instructed in CSI procedures by the senior author in six group meetings, during which extensive modeling and role-playing experiences were provided. Once the six 30 -min training sessions with the children were initiated, weekly supervision meetings occurred during the course of the study to insure that experimenters' handling of the training was proceeding appropriately and uniformly.

The training tasks ranged from a simple coloring exercise to more complex, sequentially numbered dot connection problems, hidden figure drawings, and mazes selected from a sampling of age-appropriate children's magazines. Early trials indicated that the sequentially numbered dot connection problems were most appropriate for the modeling of the relevant cognitive strategies, and consequently, the majority of the training was accomplished with the dot tasks.

For the five experimental (CSI) children, the trainer modeled appropriate task behaviors, including the verbalization of selfinstruction and self-reinforcement (praise) for corrections of errors and task completion. Selective cuing and reinforcement was then provided for the children's imitation of the trainermodeled behavior on the same tasks. Each trainer worked with one experimental (CSI) child and a matched placebo control (PC) child, who was presented with the same materials as his/her CSI counterpart but received no CSI training. An additional group of four children served as time controls (TC).

Pre- and posttreatment administrations of seven measures were used to assess CSI training effects on a wide range of skills and abilities essential to traditional academic learning. These measures included a teacher-completed rating scale (Conners, 1969) of the children's classroom behaviors (principally social in nature), as well as the Matching Familiar Figures test (MFF), a 
traditional measure of cognitive impulsivity (Kagan, 1966); the Children's Embedded Figures test (CEFT), a measure of children's selective scanning and disembedding abilities (Witkin, Oltman, Raskin, \& Karp, 1971); and the picture arrangement, block design, and coding subtests from the Wechsler Intelligence Scale for Children (WISC) (Wechsler, 1949). A final measure was provided by the prorated performance IQ derived from the three WISC subtests.

\section{RESULTS}

The pre- and posttreatment means and standard deviations for the three subject groups on the eight dependent measures are presented in Table 1. For each of the pretreatment means for the seven dependent measures, $F$ tests revealed no significant differences between the three groups prior to treatment. Repeatedmeasures ANOVAs and preplanned multiple t tests (summarized in Table 1) were used to assess the effects of treatment on each of the dependent measures.

The analysis of variance for the three groups' mean latency of decision on the pre- and posttreatment MFF tests revealed significant treatment $[\mathrm{F}(2,11)=6.18$, $\mathrm{p}<.025]$ and trials $[\mathrm{F}(1,11)=12.80, \mathrm{p}<.005]$ effects,

Table 1

Pre- and Posttreatment Means and Standard Deviations of Cognitive Self-Instruction (CSI), Placebo Control (PC), and Time Control (TC) Groups on Assessment Measures

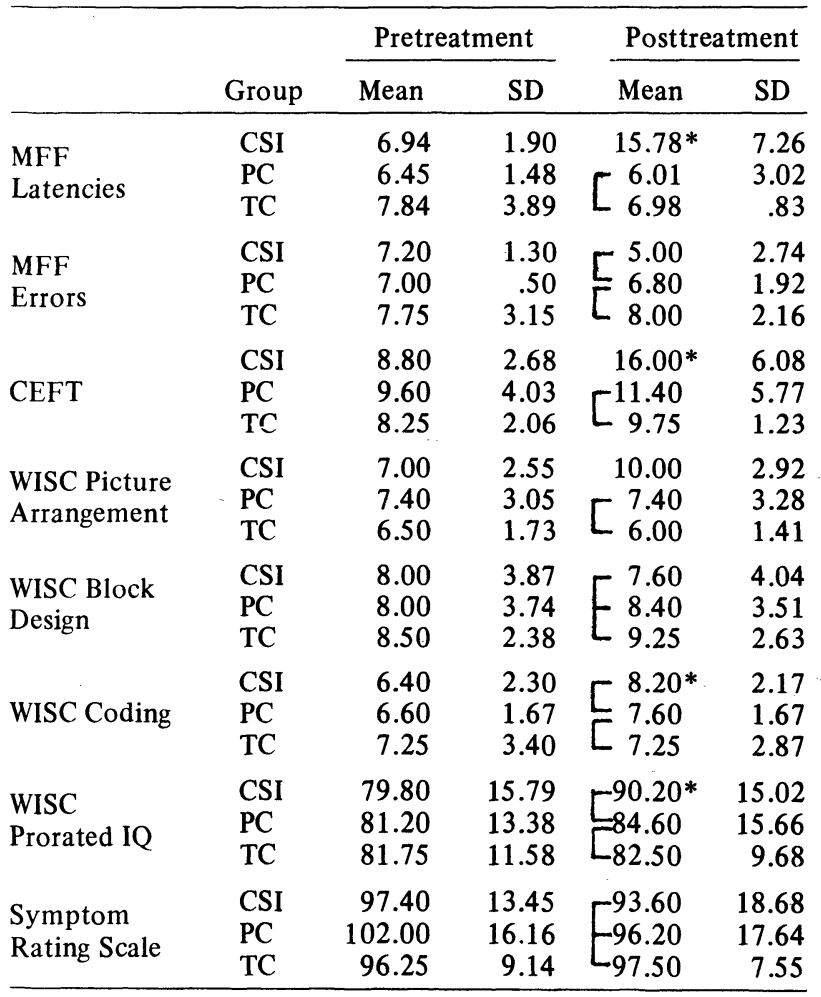

Note-For each of the dependent measures, postreatment means not connected by brackets differ at .05 level or less (two-tailed multiple $t$ comparisons). Those posttreatment means followed by an asterisk differ from the relevant pretreatment mean at .05 level or less. as well as a significant Treatment by Trials interaction $[F(2,11)=17.41, p<.005]$. Further analysis of these effects indicated that only the CSI group demonstrated a significant change in latencies across the two MFF administrations. Additionally, as evidenced by the multiple t-test comparisons among the three groups' posttreatment means, the CSI group demonstrated significantly longer latencies during the second administration of the MFF test as compared to the PC and TC control groups. The latter two groups' latencies for the posttreatment MFF test were not significantly different.

On the CEFT, a significant trials effect $[F(1,11)=$ 23.04, $\mathrm{p}<.001$ ] and a significant Treatment by Trials interaction $[F(2,11)=6.12, p<.05]$ were found. Further analyses of the trials effect and the Treatment by Trials interaction indicated that the number of forms correctly identified on the posttreatment CEFT for the CSI group was significantly greater than that for the two control groups (for both comparisons, $p<.05$ ), which did not significantly differ from each other. Additionally, the increase from the pre- to the posttreatment administration for the CSI group was significant $(p<.001)$, while no significant change from preto posttreatment CEFT scores existed for the two control groups.

Further confirmation of the efficacy of CSI training was provided by the WISC subtest changes. A significant Treatment by Trials interaction was obtained for the picture arrangement measure $[\mathrm{F}(2,11)=5.05, \mathrm{p}<.05]$, and the multiple $t$ comparisons demonstrated that the CSI group's mean score on the posttreatment picture arrangement test was significantly higher than that of the PC group $(p<.05)$ and the TC group $(p<.05)$. The latter two groups did not differ significantly on the posttreatment picture arrangement test. For the coding subtest and the prorated WISC performance IQ scores, only the trials effect was significant [coding trials effect: $F(1,11)=4.75, p<.05$; and prorated performance IQ trials effect: $F(1,11)=6.51, p<.05]$. Further analyses revealed a significant $(p<.05)$ increase on this measure only for the CSI group. Likewise, for the prorated performance IQ trials effect, only the CSI group demonstrated a significant increase $(\mathrm{p}<.05)$ on this measure across administrations. No significant changes across administrations, nor between the three groups' posttreatment scores for the block design subtest were found. Also, no significant changes in classroom behaviors as indexed by the teacheradministered behavior rating scale were found.

\section{DISCUSSION}

The obtained results, while providing strong support for the general principle of training self-mediation behaviors for hyperkinetics, also suggest limitations in the degree to which such training can be expected to generalize. Sequential dot connection tasks were the principal training task utilized, and in accordance with the requirements of this task, the processes of carefully and selectively attending to alternative choices and plan- 
ning choice behaviors were stressed. These processing skills appear most akin to those required on the MFF, CEFT, and picture arrangement and coding subtests, which resulted in significant improvement on these tasks. By way of contrast, observations of CSI subjects' behaviors during the posttreatment administration of the block design subtest suggest that they were more reflective and deliberate. However, the specifics of the trained self-instruction apparently did not provide an adequate increase in part-whole analysis skills essential for efficient block design performance, as the number of designs correctly assembled within the time limits of the test did not increase.

The lack of classroom behavioral changes noted in the present study can also most probably be attributed to the nature of the training tasks used. These tasks (sequential dot connection problems, mazes, etc.) are essentially cognitive in nature. Transfer of training effects to the complex social environment of the classroom may well depend upon the inclusion of specifically socially oriented content within the CSI training package.

In conclusion, CSI training with hyperkinetic children has excellent potential in modifying cognitive processes that are critical for successful academic performance. The effectiveness of CSI training for more general applications, such as preparing hyperkinetics more adequately for the traditional classroom setting, will probably depend upon a careful analysis of the specific situational and task requirements with which the child is encountering difficulties.

\section{REFERENCES}

Alabiso, F. Inhibitory functions of attention in reducing hyperactive behavior. American Journal of Mental Deficiency, 1972, 77, 259-282.
Campbell, S. B., Douglas, V. I., \& Morgenstern, G. Cognitive style in hyperactive children and the effect of methylphenidate. Journal of Child Psychology and Psychiatry, 1971, 12, 55-67.

Cohen, N. J., Weiss, G., \& Minde, K. Cognitive styles in adolescents previously diagnosed as hyperactive. Journal of Child Psychology and Psychiatry, 1972, 13, 203-209.

Cole, S. O., \& Moore, S. F. The hyperkinetic child syndrome: The need for reassessment. Child Psychiatry and Human Development, 1976, 7, 103-112.

Conners, C. K. A teacher rating scale for use in drug studies with children. American Journal of Psychiatry, 1969, 126, 884-888.

Douglas, V. I. Are drugs enough? To treat or to train the hyperactive child. International Journal of Mental Health, 1975, 4, 199-212.

KAGAN, J. Reflection-impulsivity: The generality and dynamics of conceptual tempo. Journal of Abnormal Psychology, 1966, 71, 17-24.

Meichenbaum, D., \& Goodman, J. Training impulsive children to talk to themselves: A means of developing self-control. Journal of Abnormal Psychology, 1971, 77, 115-126.

WECHSLER, D. Manual: Wechsler intelligence scale for children. New York: Psychological Corporation, 1949.

Witkin, H. A., Oltman, P. K., Raskin, E., \& KarP, S. $A$ manual for the embedded figures tests. Palo Alto, Calif: Consulting Psychologists Press, 1971.

(Received for publication March 6, 1978.) 